\title{
A New Process-oriented and Spatiotemporal Data Model For GIS Data
}

\author{
Shen Yawen \\ School of Remote Sensing and Information Engineering, Wuhan University, China 430079- Shen289554864@163.com
}

\author{
Commission VI, WG VI/4
}

KEY WORDS: Spatiotemporal data model, Simulation for dynamic process, Event-driven model

\begin{abstract}
:
With the rapid development of wireless sensor and information technology, there is a trend of transition from "digital monitoring " to "intelligence monitoring" advancing process. The traditional model cannot completely match the dynamic data to accurately describe changes of geographical and environmental changes. In this paper, we try to build a process-oriented and real-time spatiotemporal data model to meet the demands. With various types of monitoring devices, detection methods and the utilization of new technologies, the model can simulate the possible waterlog area in a specific year by analyzing the given data. By testing and modifying the spatiotemporal model, we can come to a rational conclusion that our model can forecast the actual situation in certain extent.
\end{abstract}

\section{INTRODUCTION}

Simulation and expression for the dynamic process of spatial and temporal geographic data have characteristics like multi origins, high variability and uneven distributions. Therefore the traditional static GIS and geographic information system is not sufficient to support the dynamic expression and the evolution of the geographical process nowadays. The demand of the storage, management, and related properties of data cannot be satisfied as well. Even worse, the expression of complex spatiotemporal process semantics cannot support multi sensors, granularities and multi level spatiotemporal changes. So in this paper, we try to propose a multi-level spatiotemporal data model with spatiotemporal process. On the basis of analyzing the existing video data model, we combine the spatial data storage, process modelling and visual technology to build a real-time expression model.

\section{MAINBODY}

\subsection{Research Contents}

This paper will focus on the general phenomenon and process of temporal geographical phenomena. Our new spatiotemporal data model combined with the needs of real-time monitoring based on GIS, spatial relationship and the model of digital objects through various spatial analysis. The core part of it is a spatiotemporal process driven by multi layer factors based on event that can be briefly described as follows: in a certain time frame, the sensor seized geographical objects produced by events. Events driven model through multi factor processing, resulting in higher level events and objects to change the geometry state. And then the higher level events promote the evolution of spatial and temporal information.

\subsection{General Situation of Research Area}

In July 2016, Wuhan, China encountered severe floods. And by the end of July 6th, 757 thousand people in the city were affected by the disaster, 97404 hectares of crops were damaged. The flood caused the direct economic loss 2 billion 265 million yuan. In order to avoid such damage in the future, we try to build a disaster simulation system which can monitor relative geological phenomena and predict the future flood. Our research is based on the conventional weather observation data and the geological data of Wuhan during 1980-2016. It has been shown that in Wuhan the precipitation in flood season is up to $93 \mathrm{~mm}$, and the frequency of extreme precipitation during flood season is on a rising trend, especially during 2015-2016.

\subsection{Technical Routes}

Our model will be based on the phenomena and the processes of floods in Wuhan, the dynamic simulation of human activities and real-time expression. To copy with different forms of changing information in these waterlog areas, we choose to use the 3D modelling technology, the management on massive data and visualization to build the preliminary model. Then, by using deductive method, the feasibility of the model can be tested.

We hope to establish a model integrating multi-source data, multi-dimensional spatiotemporal data and supporting time correlation. The model will consider the traditional GIS and spatial data management and focus on a specific geographical phenomena in a specific area.

The technical routes can be divided into four steps:

(1)Analyse the floods and the influences of human activities of past years in Wuhan, and combine existing models to build a preliminary model.

(2)By analyzing its feasibility, we design the structure of the model according to the facts and then complete the development of the prototype.

(3)On the basis of the prototype platform, we carry out the dynamic regulation of waterlogging and the detection of precipitation process.

(4)By testing the model, we modify the original model and perfect the system. 


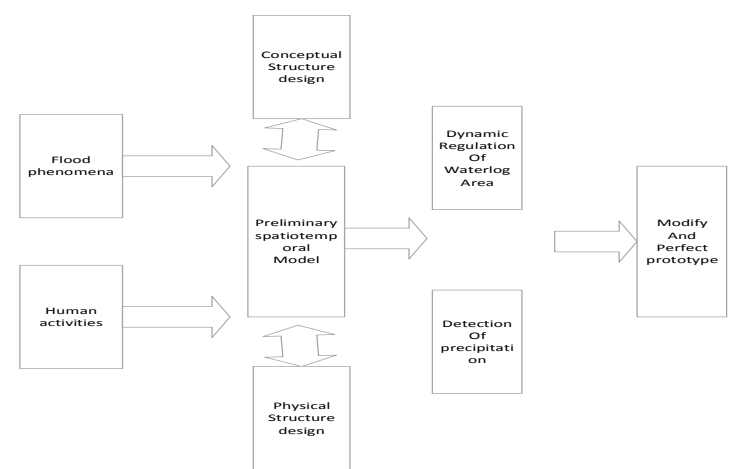

Figure 1. Technical routes of the model

\subsection{Conceptual Framework}

We use the events that contain "changing information" to describe the interactions and relationships between objects, and thus reflecting the changing process of objects and their status with time and space. The model does not only consider the management of traditional spatial data but also supports realtime visualization of management and process of multi-sensor data. It is a spatiotemporal data model for dynamic process simulation and real-time expression. The main research contents include:

Based on the analysis of the existing spatiotemporal data models, a spatiotemporal data model driven by events and multiple factors is proposed to describe the characteristics of various geographic processes.

The model presented in this work considers the dynamic development of geographical phenomena and the requirement of process monitoring and supervision, and makes use of technologies including storage management, modeling and visualization of massive spatial data. This model can be divided into four layers:

1) The spatiotemporal process modeling layer: Based on the general spatiotemporal GIS model, combined with the multi factor driving model of geographic events and the composition of agent objects, its core part of the model is expanded.

2) Geometric model layer: it provides the geometric representation of the entity objects and extends the part of the basic model.

3) Modelling and scaling layer: Based on the previous layers, a four dimensional spatiotemporal geographic model that supports multiple space-time scales is built.

4) Semantic layer expresses the temporal, spatial and geographical features supported by spatiotemporal objects.

The four layers coordinate with each other, and are built from bottom to top orderly. They can support multi-scale geospatial objects, multi level observation data, multi-level events and multi time granularities. The core part of the spatiotemporal process modelling layer is mainly composed of real-time GIS spatiotemporal data model and event multi factor driving model. Its basic concepts are described below.

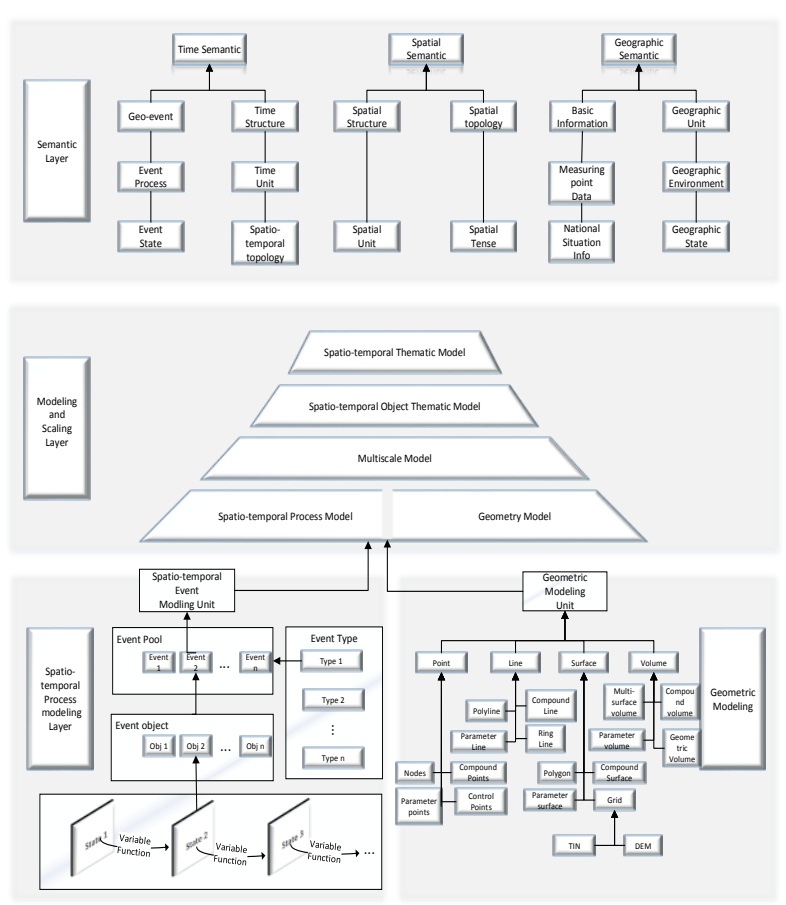

Figure 2. Geographic spatiotemporal concept model

\subsection{Application designing}

Using on the geographical spatiotemporal data model, we applied the MongoDB database to storage management model, geographical elements and related data. We also designed and implemented the dynamic monitoring, the simulation of the basic geographic information system and geologic hazards (such as landslide and fire disaster) by multi sensors. 。

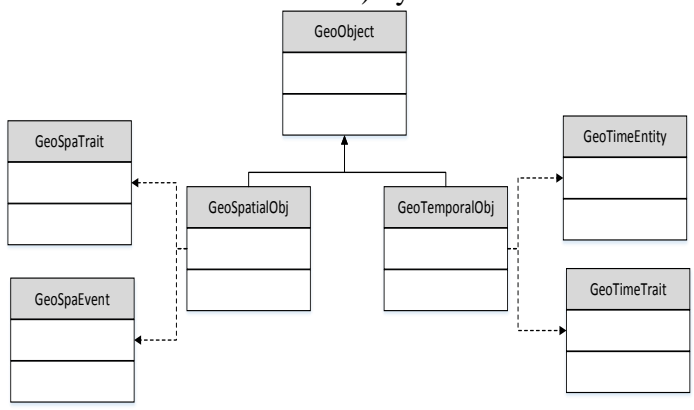

Figure 3. Spatiotemporal object concept structure

As shown in Figure 3, the GeoObject is composed of GeoSpatialObj and GeoTemporalObj. Geospatial object (GeoSpatial Object) is a combined object class that contains the Geo-traits, and Geospatial events. The former one describes the geometry, texture, material and multi-detail levels of geographic features. The latter one describes the spatial reference system information.

GeoTemporalObj (Geo-time object) is a combined object class that contains the geo-time entity and the geo-time trait. The former one describes information such as time, date, geochronology, and time interval or time period. And the latter one describes the time reference system information. The time axis is represented by both of them. GeoTimeEntity expresses a specific part of the timeline. The time domain is defined by GeoTimeTrait. And the value on the timeline is defined by GeoTimeEntity. 


\begin{tabular}{|l|l|l|l|l|}
\hline EventID & Name & Type & Trait & LinkEventID \\
\hline String & String & Int & String & Int \\
\hline CalculateTime & Action & ID & Event & SubEventID \\
\hline Int & String & String & $\begin{array}{l}\text { Bindat } \\
\text { a }\end{array}$ & Bindata \\
\hline
\end{tabular}

Figure 4. Fields structures

During the designing process of application system, we adapt several different methods to calculate the modulus of drainage which is very important for the model to exact simulate the possible waterlog area. The modulus of drainage is the maximum drainage per unit area. Here is the main method we used in the model to get the outflows in a certain region.

Horton model: a nonlinear method consisting of two equations to calculate the drainage in each sub-region.

$$
\left\{\begin{array}{c}
Q=\varpi \frac{1.49}{n}\left(h-h_{p}\right)^{5 / 3} S^{1 / 2} \\
A \frac{d h}{d t}=A(i-f)-Q
\end{array}\right.
$$

Where

$$
\begin{aligned}
& \mathrm{Q}=\text { outflows of the sub-regions, } \mathrm{m}^{3} / \mathrm{s} \\
& \mathrm{A}=\text { the area of regions, } \mathrm{hm}^{2} \\
& \omega=\text { characteristic width of the region, } \mathrm{m} \\
& \mathrm{i}=\text { the rainfall intensity, } \mathrm{mm} / \mathrm{h} \\
& \mathrm{f}=\text { flow rate, } \mathrm{mm} / \mathrm{h} \\
& \mathrm{S}=\text { flood slope, } \% \\
& \mathrm{~h}=\text { average depth of surface runoffs, } \mathrm{mm} \\
& \mathrm{h}_{\mathrm{p}=} \text { depth of detained water, } \mathrm{mm} \\
& \mathrm{n}=\text { manning coefficient }
\end{aligned}
$$

\section{CONCLUSIONS}

On the basis of new spatiotemporal model, we use the previous rainfall data of Wuhan and its geological information to simulate the possible distribution of waterlog disaster. Figure 3 is the thermogram of the possible zone, which rainfall are greater than the annual average number in 2017 , calculated by our model based on the information of 2010-2016. And the figure 5 is the actual zone in 2017.

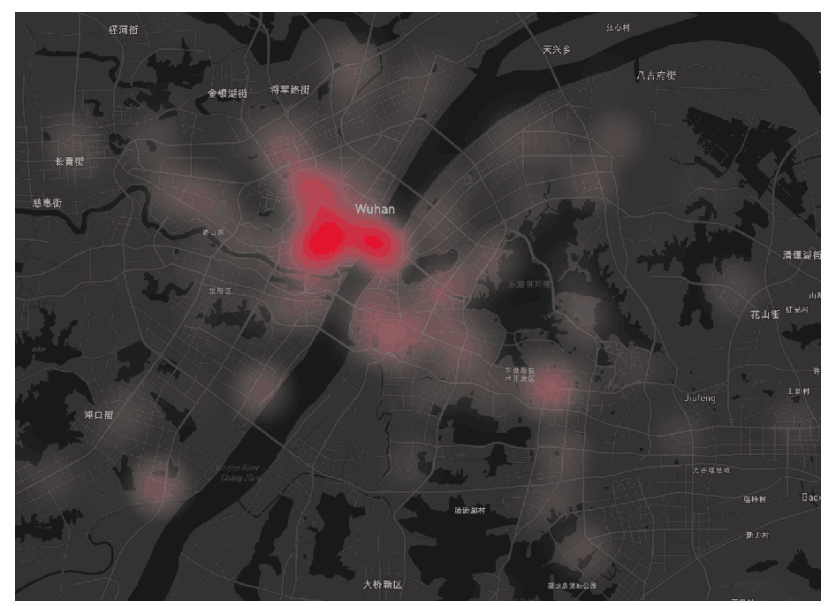

Figure 3. Thermogram of possible waterlog zone in 2017

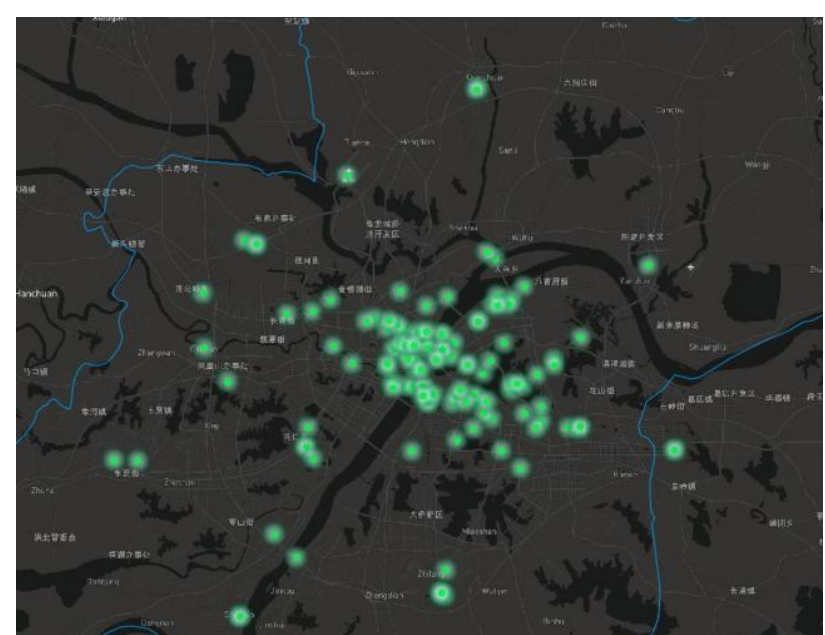

Figure 4. Actual waterlog zone in 2017

By comparing these two figures, we can see that through research, test, and application, the design of the geographic data model, focusing on simulation of the process and real-time expression of time and location, is feasible, reasonable and effective. The model is compatible with traditional GIS and spatio-temporal data information system, and also has the ability to make full use of the spatial database that was previously stored. Moreover, the design can be implemented to realize the application system through the interfaces among distributed and non-relational databases. The model can support the partition of data and the management of the cluster, incorporate data from multiple sources, different spatial and temporal domains to satisfy the increasing needs of managing multi-dimensional data.

\section{REFERENCES}

Guttman A. R-trees: a dynamic index structure for spatial searching[M]. ACM, 1984.

Lin H. Efficient and compact indexing structure for processing of spatial queries in line-based databases[J[. Data \& Knowledge Engineering, 2008,64(1):365-380.

Davis E, Morgenstern L. Introduction: Progress in formal commonsense reasoning[J]. Artificial Intelligence, 2004,153(1): $1-12$

Andrienko N, Andrienko G, Gatalsky P. Exploratory spatiotemporal visualization: an analytical review $[\mathrm{J}]$. Journal of Visual Languages \& Computing, 2003,14(6):503-541.

Chomicki J, Liu Y, Revesz P. Animating spatiotemporal constraint databases[A], In: Spatio-Temporal Database Management, 1999[C]. Springer. 